\title{
Commentary
}

\section{The Physiological Deadlock between AMPK and Gluconeogenesis}

\section{SOGA, a Novel Protein, May Provide the Key}

\author{
Josephine M. Forbes \\ From the Diabetes Division, Baker IDI Heart and Diabetes \\ Institute, and the Department of Immunology, Monash \\ University, Melbourne, Australia
}

Numerous chronic disorders directly result from metabolic imbalances, including obesity and insulin resistance, which are major risk factors for the development of type 2 diabetes, the largest global epidemic of the 21st century to date. Evolution has provided us with a biological sensor for systemic energy balance, adenosine monophosphate kinase (AMPK). AMPK integrates nutritional and hormonal signals in peripheral tissues and the hypothalamus by mediating the effects of adipokines, such as adiponectin, to regulate food intake, body weight, and glucose and lipid homeostasis. ${ }^{1}$ There is a paradox, however, that surrounds the actions of insulin and adiponectin on gluconeogenesis in the liver, elegantly alluded to in this issue of the journal. Cowerd et $\mathrm{al}^{2}$ present a convincing case that the actions of a novel protein, suppressor of glucose by autophagy (SOGA), can explain how adiponectin leads to insulin-dependent inhibition of autophagy during the activation of AMPK. Autophagy in the liver provides substrates for gluconeogenesis via lysosomal breakdown of cellular proteins, glycogen, and triglycerides. Indeed, autophagy is stimulated by AMPK activation. In insulin resistance, hyperinsulinemia, in the context of a decline in circulating adiponectin, eventually leads to a loss of adequate hepatic insulin signaling and enhanced gluconeogenesis in the liver. One is at a loss to explain why autophagy is increased by insulin resistance, which contributes to enhanced gluconeogenesis. Interestingly, Cowerd et $\mathrm{al}^{2}$ hypothesized that there may be a loss of the expression of the biological "brake" for autophagy in the liver, the newly discovered SOGA protein, under conditions such as insulin resistance. Hence, they discovered a novel therapeutic target for this disorder because the elevation of SOGA (ie, in response to adiponectin) is able to lower liver glucose production through the inhibition of autophagy, which results in a decrease in plasma glucose concentrations.
Insulin resistance is broadly characterized by an inability of target tissues to respond to normal levels of the endocrine pancreatic hormone, insulin. The liver, skeletal muscle, and adipose tissues are the most insulin-sensitive tissues because of their large capacity for use and storage of glucose, amino acids, and lipids on activation of insulin signaling pathways. Skeletal muscle accounts for approximately $80 \%$ of glucose disposal in the presence of insulin ${ }^{3}$ and thus represents the primary site for insulin resistance. Indeed, the rate of insulin-stimulated glucose uptake by muscle is $60 \%$ lower in insulin-resistant individuals than in insulin-sensitive controls. ${ }^{4}$ At rest, skeletal muscle depends primarily on fatty acids for energy, although this energy source rapidly switches to glucose after meals and during exercise. With insufficient glucose uptake and glycogen stores, insulin resistance in the muscle limits the energy available to the muscle during exercise (eg, when the muscle depends on glucose), resulting in an $80 \%$ increase in intramyocellular lipid content ${ }^{4}$ and poor exercise performance. ${ }^{3}$

Within the liver, insulin either promotes the uptake of glucose and its conversion to glycogen for local storage or signals for fatty acids to be transported to adipose tissue. Insulin also acts on the liver to decelerate the conversion of glycogen to glucose (ie, glycogenolysis). In addition to the facilitation of glycogenolysis, insulin resistance in the liver is associated with sustained gluconeogenesis (ie, glucose formation from precursors other than glycogen, such as D-lactate), which further contributes to elevated blood glucose levels. ${ }^{5}$ It is widely understood that sustained gluconeogenesis is a hallmark of insulin resistance and a major risk factor for type 2 diabetes.

Accepted for publication July 7, 2010.

CME Disclosure: The author did not disclose any relevant financial relationships.

Address reprint requests to Josephine Forbes, Ph.D., Glycation and Diabetes, Baker IDI Heart and Diabetes Institute, P.O. Box 6492, St. Kilda Rd. Central, Melbourne, VIC, 8008 Australia. E-mail: josephine.forbes@ bakeridi.edu.au. 
Within their studies, Cowerd et $\mathrm{al}^{2}$ showed that knockdown of their newly discovered SOGA protein in liver cells results in increased autophagy, providing substrates for gluconeogenesis.

Glucose production in the liver depends on autophagy. Autophagy, or autophagocytosis, is a tightly regulated catabolic process that involves the degradation of a cell's own components via lysosomal machinery. Autophagy provides the biochemical intermediates for glucose production through the hydrolysis of proteins, glycogen, and triglycerides. ${ }^{6,7}$ It is a major mechanism by which a starving cell reallocates nutrients from unnecessary processes to processes that are more essential. The most well characterized process leading to autophagy involves the formation of a membrane around a targeted region of the cell, separating the contents from the rest of the cytoplasm. The resultant vesicle then fuses with a lysosome, which subsequently degrades the vesicular contents. Although autophagy was first described in the $1960 \mathrm{~s},{ }^{8}$ its role in disease is not well categorized. Indeed, autophagy may either assist or halt the progression of certain diseases, such as some types of neurodegeneration and cancer ${ }^{9}$ and play a protective role against infection by intracellular pathogens. However, in some situations, it may actually contribute to the development of a disease.

During nutrient starvation, increased levels of autophagy lead to the breakdown of nonvital components and the release of nutrients, ensuring that vital processes can continue. ${ }^{10}$ Not surprisingly, mutant yeast cells that have a reduced autophagic capability rapidly perish under nutrition-deficient conditions. ${ }^{11}$ Because insulin inhibits autophagy, conditions in which there is a deficiency in insulin, such as fasting, starvation, and type I diabetes, increase autophagy in the liver. ${ }^{12,13}$ Indeed, in the studies presented in the American Journal of Pathology this month, wild-type mice that underwent caloric restriction had a doubling of serum adiponectin concentrations, an adipocyte-secreted molecule that circulates under conditions that increase SOGA protein levels.

It is interesting that increasing adiponectin concentrations using pioglitazone in ob/ob mice in the studies of Cowerd et al also had profound effects on liver SOGA expression. These results support the hypothesis that the elevation of SOGA levels by adiponectin increases insulin sensitivity, again highlighting the fact that elevations in hepatic SOGA concentrations may impair gluconeogenesis in insulin-resistant and type 2 diabetic individuals, thereby improving their insulin sensitivity by lowering circulating glucose concentrations. Furthermore, certain diets designed for weight loss in humans use a form of autophagy. Indeed, the Atkins diet relies heavily on ketosis as a method of reducing body fat, which, in itself, can be considered a form of cellular autophagy. It is tempting to speculate that the success of this type of diet in improving insulin sensitivity may be via the direct inhibition of autophagy in the liver, thus decreasing gluconeogenesis.

It is also interesting, however, that exposure to excess insulin, as would be seen in insulin resistance, has been shown to decrease liver autophagy via activation of
mTOR, a serine/threonine protein kinase that integrates input from multiple cellular pathways. ${ }^{14}$ This finding is most likely the result of a reduction in hepatic insulin signaling after long-term hyperinsulinemia. If this is the case, however, where are the substrates for the excessive gluconeogenesis that occurs derived from under these circumstances? Cowerd et $\mathrm{al}^{2}$ stated that they were "perplexed by reports that AMPK, an essential mediator of adiponectin action [which include increasing hepatic fatty acid and glucose uptake and inhibition of gluconeogenesis ${ }^{15}$ ], inhibits mTOR and stimulates autophagy." ${ }^{16-19}$ However, in liver, AMPK activation is also necessary for the effects of phosphoenolpyruvate carboxykinase and glucose 6-phosphatase expression as well as for hepatic glucose production. ${ }^{20}$ Furthermore, blocking the actions of adiponectin on AMPK in liver using a dominant-negative AMPK abrogates the glucose-lowering effect of adiponectin. ${ }^{20}$ Therefore, the stimulation of AMPK phosphorylation and activity in liver by adiponectin in vivo and in vitro ${ }^{21}$ should hypothetically lead to an inhibition of autophagy. It is widely recognized that adiponectin can improve insulin sensitivity via the activation of hepatic AMPK, which reduces the gene expression levels of rate-limiting enzymes for glucose production. ${ }^{20,22,23}$

Overall, Cowerd et al suggested that there might be a loss of the expression of the biological "brake" for autophagy in the liver, the newly discovered SOGA protein, under conditions such as insulin resistance, in which the concentrations of circulating adiponectin are decreased. In addition, they hypothesize that the opposite is also true: there is an increase in SOGA expression under conditions in which increased adiponectin concentrations, associated with caloric restriction and ketosis (ie, conditions of starvation and weight loss), are protective via a decline in hepatic autophagy. Hypothetically, their discovery could have implications not only for insulin resistance but also for obesity, weight loss, and nutritional deficiencies, which could all be improved via stimulation of hepatic SOGA expression. Circulating fragments of the SOGA protein may also find utility as biomarkers for hepatic SOGA concentrations, warranting further investigation in larger human cohorts.

\section{References}

1. Kahn BB, Alquier T, Carling D, Hardie DG: AMP-activated protein kinase: ancient energy gauge provides clues to modern understanding of metabolism. Cell Metab 2005, 1:15-25

2. Cowerd RB, Asmar MM, Alderman JM, Alderman EA, Garland AL, Busby WH, Bodnar WM, Rusyn I, Medoff BD, Tisch R, Swenberg JA Zeisel SH, Combs TP: Adiponectin lowers glucose production by increasing SOGA. Am J Pathol 2010, 177:1936-1945

3. Kiens B: Skeletal muscle lipid metabolism in exercise and insulin resistance. Physiol Rev 2006, 86:205-243

4. Petersen KF, Dufour S, Befroy D, Garcia R, Shulman GI: Impaired mitochondrial activity in the insulin-resistant offspring of patients with type 2 diabetes. N Engl J Med 2004, 350:664-671

5. Bouche C, Serdy S, Kahn CR, Goldfine AB: The cellular fate of glucose and its relevance in type 2 diabetes. Endocr Rev 2004, 25:807-830

6. Mortimore GE, Poso AR: Intracellular protein catabolism and its con- 
trol during nutrient deprivation and supply. Annu Rev Nutr 1987, 7:539-564

7. Singh R, Kaushik S, Wang Y, Xiang Y, Novak I, Komatsu M, Tanaka K, Cuervo AM, Czaja MJ: Autophagy regulates lipid metabolism. Nature 2009, 458:1131-1135

8. Kundu M, Thompson CB: Autophagy: basic principles and relevance to disease. Annu Rev Pathol 2008, 3:427-455

9. Mizushima N, Klionsky DJ: Protein turnover via autophagy: implications for metabolism. Annu Rev Nutr 2007, 27:19-40

10. Yorimitsu T, Klionsky DJ: Autophagy: molecular machinery for selfeating, Cell Death Differ 2005, 12(Suppl 2):1542-1552

11. Tsukada M, Ohsumi Y: Isolation and characterization of autophagydefective mutants of Saccharomyces cerevisiae. FEBS Lett 1993, 333:169-174

12. Amherdt M, Harris V, Renold AE, Orci L, Unger RH: Hepatic autography in uncontrolled experimental diabetes and its relationships to insulin and glucagon. J Clin Invest 1974, 54:188-193

13. de Waal EJ, Vreeling-Sindelarova H, Schellens JP, Houtkooper JM, James J: Quantitative changes in the lysosomal vacuolar system of rat hepatocytes during short-term starvation. A morphometric analysis with special reference to macro- and microautophagy, Cell Tissue Res 1986, 243:641-648

14. Kanazawa T, Taneike I, Akaishi R, Yoshizawa F, Furuya N, Fujimura S, Kadowaki M: Amino acids and insulin control autophagic proteolysis through different signaling pathways in relation to MTOR in isolated rat hepatocytes. J Biol Chem 2004, 279:8452-8459

15. Goldstein BJ, Scalia R: Adiponectin: a novel adipokine linking adipocytes and vascular function. J Clin Endocrinol Metab 2004, 89: 2563-2568

16. Cheng SW, Fryer LG, Carling D, Shepherd PR: Thr2446 is a novel mammalian target of rapamycin (mTOR) phosphorylation site regulated by nutrient status. J Biol Chem 2004, 279:15719-15722

17. Liang J, Shao SH, Xu ZX, Hennessy B, Ding Z, Larrea M, Kondo S, Dumont DJ, Gutterman JU, Walker CL, Slingerland JM, Mills GB: The energy sensing LKB1-AMPK pathway regulates p27 $7^{\mathrm{kip} 1}$ phosphorylation mediating the decision to enter autophagy or apoptosis. Nat Cell Biol 2007, 9:218-224

18. Meijer AJ, Codogno P: AMP-activated protein kinase and autophagy. Autophagy 2007, 3:238-240

19. Meley D, Bauvy C, Houben-Weerts JH, Dubbelhuis PF, Helmond MT, Codogno P, Meijer AJ: AMP-activated protein kinase and the regulation of autophagic proteolysis. J Biol Chem 2006, 281:34870-34879

20. Yamauchi T, Kamon J, Minokoshi $Y$, Ito $Y$, Waki H, Uchida S, Yamashita S, Noda M, Kita S, Ueki K, Eto K, Akanuma Y, Froguel P, Foufelle F, Ferre P, Carling D, Kimura S, Nagai R, Kahn BB, Kadowaki $\mathrm{T}$ : Adiponectin stimulates glucose utilization and fatty-acid oxidation by activating AMP-activated protein kinase. Nat Med 2002, 8:1288-1295

21. Minokoshi $Y$, Alquier T, Furukawa N, Kim YB, Lee A, Xue B, Mu J, Foufelle F, Ferre P, Birnbaum MJ, Stuck BJ, Kahn BB: AMP-kinase regulates food intake by responding to hormonal and nutrient signals in the hypothalamus. Nature 2004, 428:569-574

22. Combs TP, Berg AH, Obici S, Scherer PE, Rossetti L: Endogenous glucose production is inhibited by the adipose-derived protein Acrp30. J Clin Invest 2001, 108:1875-1881

23. Tomas E, Tsao TS, Saha AK, Murrey HE, Zhang Cc, Itani SI, Lodish HF, Ruderman NB: Enhanced muscle fat oxidation and glucose transport by ACRP30 globular domain: acetyl-CoA carboxylase inhibition and AMP-activated protein kinase activation. Proc Natl Acad Sci USA 2002, 99:16309-16313 